\title{
On differential subordinations in the complex plane
}

\author{
Mamoru Nunokawa ${ }^{1}$, Nak Eun Cho ${ }^{2 *}$, Oh Sang Kwon ${ }^{3}$ and Janusz Sokół ${ }^{4}$
}

${ }^{\text {*Correspondence: }}$

necho@pknu.ac.kr

${ }^{2}$ Department of Applied

Mathematics, College of Natural

Sciences, Pukyong National

University, Pusan, 608-737, Korea

Full list of author information is

available at the end of the article

\begin{abstract}
The purpose of this work is to present a new approach to solve some problems in differential subordination theory. We also discuss the new results closely related to the generalized Briot-Bouquet differential subordination.
\end{abstract}

MSC: Primary 30C45; secondary 30C 80

Keywords: analytic functions; convex functions; convex of order alpha; univalent functions; differential subordination

\section{Introduction}

Let $\mathcal{H}$ denote the class of all analytic functions in the unit disc $\mathbb{D}=\{z:|z|<1\}$ in the complex plane $\mathbb{C}$. Recall that a set $E \subset \mathbb{C}$ is said to be starlike with respect to a point $w_{0} \in E$ if and only if the linear segment joining $w_{0}$ to every other point $w \in E$ lies entirely in $E$, while a set $E$ is said to be convex if and only if it is starlike with respect to each of its points, that is, if and only if the linear segment joining any two points of $E$ lies entirely in $E$. A univalent function $f$ maps $\mathbb{D}$ onto a convex domain $E$ if and only if [1]

$$
\mathfrak{R e}\left\{1+\frac{z f^{\prime \prime}(z)}{f^{\prime}(z)}\right\}>0 \quad \text { for all } z \in \mathbb{D}
$$

and then $f$ is said to be convex in $\mathbb{D}$ (or briefly convex). Let $\mathcal{A}$ denote the subclass of $\mathcal{H}$ consisting of functions normalized by $f(0)=0, f^{\prime}(0)=1$. We denote by $\mathcal{K}$ the set of all functions $f \in \mathcal{A}$ that are convex univalent in $\mathbb{D}$. We say that $f \in \mathcal{A}$ is convex of order $\alpha$, $0 \leq \alpha<1$, when

$$
\mathfrak{R e}\left\{1+\frac{z f^{\prime \prime}(z)}{f^{\prime}(z)}\right\}>\alpha \quad \text { for all } z \in \mathbb{D} \text {. }
$$

Functions that are convex of order $\alpha$ were introduced by Robertson in [2]. For two analytic functions $f$, $g$, we say that $f$ is subordinate to $g$, written as $f \prec g$, if and only if there exists an analytic function $\omega$ with property $|\omega(z)| \leq|z|$ in $\mathbb{D}$ such that $f(z)=g(\omega(z))$. In particular, if $g$ is univalent in $\mathbb{D}$, then we have the following equivalence:

$$
f(z) \prec g(z) \quad \Longleftrightarrow \quad f(0)=g(0) \text { and } f(\mathbb{D}) \subset g(\mathbb{D}) \text {. }
$$

The idea of subordination was used for defining many of classes of functions studied in geometric function theory. For obtaining the main result, we shall use the methods of

(c) 2015 Nunokawa et al.; licensee Springer. This is an Open Access article distributed under the terms of the Creative Commons Attribution License (http://creativecommons.org/licenses/by/4.0), which permits unrestricted use, distribution, and reproduction in any medium, provided the original work is properly credited. 
differential subordinations. The main results in the theory of differential subordinations were introduced by Miller and Mocanu in [3] and [4]. A function $p$, analytic in $\mathbb{D}$, is said to satisfy a first-order differential subordination if

$$
\phi\left(p(z), z p^{\prime}(z)\right) \prec h(z)
$$

where $\left(p(z), z p^{\prime}(z)\right) \in D \subset \mathbb{C}^{2}$ for $z \in \mathbb{D}, \phi: \mathbb{C}^{2} \rightarrow \mathbb{C}$ and $\phi\left(p(z), z p^{\prime}(z)\right)$ is analytic in $\mathbb{D}$, and $h$ is analytic and univalent in $\mathbb{D}$. The function $q$ is said to be a dominant of the differential subordination (1.4) if $p \prec q$ for all $p$ satisfying (1.4). If $\tilde{q}$ is a dominant of (1.4) and $\tilde{q} \prec q$ for all dominants $q$ of (1.4), then we say that $\tilde{q}$ is the best dominant of the differential subordination (1.4).

The purpose of the present paper is to investigate interesting new results in connection with differential subordination and to improve some results obtained by Miller and Mocanu [5]. Also we remark that the reader may refer to the recent results obtained by Sokół and Nunokawa [6] as applications of differential subordination.

The following lemma will be required in our present investigation.

Lemma 1.1 ([3], [4, p.24]) Assume that $\mathcal{Q}$ is the set of functions $f \in \mathcal{H}$ that are injective on $\overline{\mathbb{D}} \backslash E(f)$, where

$$
E(f):=\left\{\zeta: \zeta \in \partial \mathbb{D} \text { and } \lim _{z \rightarrow \zeta} f(z)=\infty\right\},
$$

and are such that

$$
f^{\prime}(\zeta) \neq 0 \quad(\zeta \in \partial(\mathbb{D}) \backslash E(f))
$$

Let $\psi \in \mathcal{Q}$ with $\psi(0)=a$, and let

$$
\varphi(z)=a+a_{m} z^{m}+\cdots
$$

be analytic in $\mathbb{D}$ with

$$
\varphi(z) \not \equiv a \quad \text { and } \quad m \in \mathbb{N} .
$$

If $\varphi \nprec \psi$ in $\mathbb{D}$, then there exist points

$$
z_{0}=r_{0} e^{i \theta} \in \mathbb{D} \text { and } \zeta_{0} \in \partial \mathbb{D} \backslash E(\psi)
$$

for which

$$
\begin{aligned}
& \varphi\left(|z|<r_{0}\right) \subset \psi(\mathbb{D}), \\
& \varphi\left(z_{0}\right)=\psi\left(\zeta_{0}\right)
\end{aligned}
$$

and

$$
z_{0} \varphi^{\prime}\left(z_{0}\right)=s \zeta_{0} \psi^{\prime}\left(\zeta_{0}\right)
$$


for some $s \geq m$. Moreover,

$$
\mathfrak{R e}\left\{\frac{z_{0} \varphi^{\prime \prime}\left(z_{0}\right)}{\varphi^{\prime}\left(z_{0}\right)}+1\right\} \geq s \mathfrak{R e}\left\{\frac{\zeta_{0} \psi^{\prime \prime}\left(\zeta_{0}\right)}{\psi^{\prime}\left(\zeta_{0}\right)}+1\right\} .
$$

To prove the main results, we also need the following lemma, which is a generalization of a result due to Nunokawa $[7,8]$.

Lemma 1.2 Let $p(z)$ be a function analytic in $z \in \mathbb{D}$ of the form

$$
p(z)=1+\sum_{n=m}^{\infty} c_{n} z^{n}, \quad c_{m} \neq 0,
$$

with $p(z) \neq 0$ in $|z|<1$. If there exists a point $z_{0} \in \mathbb{D}$ such that

$$
|\arg \{p(z)\}|<\frac{\pi \varphi}{2} \text { for }|z|<\left|z_{0}\right|
$$

and

$$
\left|\arg \left\{p\left(z_{0}\right)\right\}\right|=\frac{\pi \varphi}{2}
$$

for some $\varphi>0$, then we have

$$
\frac{z_{0} p^{\prime}\left(z_{0}\right)}{p\left(z_{0}\right)}=i \ell \varphi
$$

where

$$
\ell \geq \frac{m}{2}\left(a+\frac{1}{a}\right) \geq m \quad \text { when } \arg \left\{p\left(z_{0}\right)\right\}=\frac{\pi \varphi}{2}
$$

and

$$
\ell \leq-\frac{m}{2}\left(a+\frac{1}{a}\right) \leq-m \quad \text { when } \arg \left\{p\left(z_{0}\right)\right\}=-\frac{\pi \varphi}{2}
$$

where

$$
\left\{p\left(z_{0}\right)\right\}^{1 / \varphi}= \pm i a \text { and } a>0 .
$$

\section{Main results}

Theorem 2.1 Let $B(z)$ and $C(z)$ be analytic in $\mathbb{D}$ with

$$
|\mathfrak{I m}\{C(z)\}|<\mathfrak{R e}\{B(z)\}
$$

If $p(z)$ is analytic in $\mathbb{D}$ with $p(0)=1$, and if

$$
\left|\arg \left\{B(z) z p^{\prime}(z)+C(z) p(z)\right\}\right|<\frac{\pi}{2}+t(z),
$$


where

$$
t(z)= \begin{cases}\arg \{B(z) i+C(z)\} & \text { when } \arg \{B(z) i+C(z)\} \in[0, \pi / 2] \\ \arg \{B(z) i+C(z)\}-\pi / 2 & \text { when } \arg \{B(z) i+C(z)\} \in(\pi / 2, \pi]\end{cases}
$$

then we have

$$
\mathfrak{R e}\{p(z)\}>0, \quad z \in \mathbb{D} \text {. }
$$

Proof By Lemma 1.2, if $\mathfrak{R e}\{p(z)\}>0$ does not hold for all $z \in \mathbb{D}$, then there exists a point $z_{0},\left|z_{0}\right|<1$, such that

$$
|\arg \{p(z)\}|<\frac{\pi}{2} \quad \text { for }|z|<\left|z_{0}\right|
$$

and

$$
\left|\arg \left\{p\left(z_{0}\right)\right\}\right|=\frac{\pi}{2}
$$

and

$$
\frac{z_{0} p^{\prime}\left(z_{0}\right)}{p\left(z_{0}\right)}=i \ell
$$

where

$$
\ell \geq \frac{1}{2}\left(a+\frac{1}{a}\right) \geq 1 \quad \text { when } \arg \left\{p\left(z_{0}\right)\right\}=\frac{\pi}{2}
$$

and

$$
\ell \leq-\frac{1}{2}\left(a+\frac{1}{a}\right) \leq-1 \quad \text { when } \arg \left\{p\left(z_{0}\right)\right\}=-\frac{\pi}{2},
$$

where

$$
p\left(z_{0}\right)= \pm i a \quad \text { and } \quad a>0 .
$$

For the case $p\left(z_{0}\right)=i a, a>0$, we are going to show that

$$
\left|\arg \left\{B\left(z_{0}\right) z_{0} p^{\prime}\left(z_{0}\right)+C\left(z_{0}\right) p\left(z_{0}\right)\right\}\right| \geq \frac{\pi}{2}+t\left(z_{0}\right) .
$$

We have

$$
\begin{aligned}
& \left|\arg \left\{B\left(z_{0}\right) z_{0} p^{\prime}\left(z_{0}\right)+C\left(z_{0}\right) p\left(z_{0}\right)\right\}\right| \\
& \quad=\left|\arg \left\{p\left(z_{0}\right)\left[B\left(z_{0}\right) \frac{z_{0} p^{\prime}\left(z_{0}\right)}{p\left(z_{0}\right)}+C\left(z_{0}\right)\right]\right\}\right| \\
& \quad=\left|\arg \left\{p\left(z_{0}\right)\left[B\left(z_{0}\right) i \ell+C\left(z_{0}\right)\right]\right\}\right| .
\end{aligned}
$$


By (2.1), we have $\mathfrak{I m}\left\{B\left(z_{0}\right) i \ell+C\left(z_{0}\right)\right\}>0$. Therefore, from (2.7) we obtain

$$
\begin{aligned}
& \left|\arg \left\{B\left(z_{0}\right) z_{0} p^{\prime}\left(z_{0}\right)+C\left(z_{0}\right) p\left(z_{0}\right)\right\}\right| \\
& \quad=\frac{\pi}{2}+ \begin{cases}\arg \{B(z) i l+C(z)\} & \text { when } \arg \{B(z) i l+C(z)\} \in[0, \pi / 2], \\
\arg \{B(z) i l+C(z)\}-\pi / 2 & \text { when } \arg \{B(z) i l+C(z)\} \in(\pi / 2, \pi]\end{cases} \\
& \quad \geq \frac{\pi}{2}+ \begin{cases}\arg \{B(z) i+C(z)\} & \text { when } \arg \{B(z) i+C(z)\} \in[0, \pi / 2], \\
\arg \{B(z) i+C(z)\}-\pi / 2 & \text { when } \arg \{B(z) i+C(z)\} \in(\pi / 2, \pi]\end{cases} \\
& \quad=\frac{\pi}{2}+t\left(z_{0}\right) .
\end{aligned}
$$

This contradicts (2.2). For the case $p\left(z_{0}\right)=-i a, a>0$, the proof runs as in the first case.

Remark 2.1 Theorem 2.1 improves a result obtained by Miller and Mocanu [5, p.208].

Corollary 2.2 Let $g(z)$ be analytic in $\mathbb{D}$ with $g(0)=1$ and $\left|\mathfrak{I m}\left\{z g^{\prime}(z) / g(z)\right\}\right|<1$. If $f(z)=$ $z+\cdots$ is analytic in $\mathbb{D}$ and

$$
\left|\arg \left\{g(z) f^{\prime}(z)\right\}\right|<\frac{\pi}{2}+v(z), \quad z \in \mathbb{D}
$$

where

$$
v(z)= \begin{cases}\arg \left\{i+1-z g^{\prime}(z) / g(z)\right\} & \text { when } \arg \left\{i+1-z g^{\prime}(z) / g(z)\right\} \in[0, \pi / 2], \\ \arg \left\{i+1-z g^{\prime}(z) / g(z)\right\}-\pi / 2 & \text { when } \arg \left\{i+1-z g^{\prime}(z) / g(z)\right\} \in(\pi / 2, \pi]\end{cases}
$$

then we have

$$
\mathfrak{R e}\left\{\frac{g(z) f(z)}{z}\right\}>0, \quad z \in \mathbb{D} .
$$

Proof We put $B(z)=1, C(z)=1-z g^{\prime}(z) / g(z), p(z)=g(z) f(z) / z$. Then $p(z)$ is analytic in $\mathbb{D}$, $p(0)=1$ and

$$
|\mathfrak{I m}\{C(z)\}|<\mathfrak{R e}\{B(z)\}=1
$$

Moreover, (2.2) becomes

$$
\left|\arg \left\{g(z) f^{\prime}(z)\right\}\right|<\frac{\pi}{2}+v(z), \quad z \in \mathbb{D} .
$$

Hence, applying Theorem 2.1, we obtain (2.9) immediately.

Theorem 2.3 Let $B(z)$ and $C(z)$ be analytic in $\mathbb{D}$ with

$$
\mathfrak{R e}\left\{\frac{C(z)}{B(z)}\right\} \geq-1, \quad z \in \mathbb{D} \text {. }
$$

If $p(z)$ is analytic in $\mathbb{D}$ with $p(0)=0$, and if

$$
\left|B(z) z p^{\prime}(z)+C(z) p(z)\right|<|B(z)+C(z)|, \quad z \in \mathbb{D},
$$


then we have

$$
|p(z)|<1, \quad z \in \mathbb{D} \text {. }
$$

Proof By Lemma 1.1, if $p(z) \nprec z$ in $\mathbb{D}$, then there exist points

$$
z_{0}=r_{0} e^{i \theta} \in \mathbb{D} \quad \text { and } \quad \zeta_{0}, \quad\left|\zeta_{0}\right|=1
$$

for which

$$
p\left(|z|<r_{0}\right) \subset \mathbb{D}, \quad p\left(z_{0}\right)=\zeta_{0}
$$

and

$$
z_{0} p^{\prime}\left(z_{0}\right)=s \zeta_{0}
$$

for some $s \geq 1$. Then, by (2.10), we have

$$
\begin{aligned}
\left|B\left(z_{0}\right) z_{0} p^{\prime}\left(z_{0}\right)+C\left(z_{0}\right) p\left(z_{0}\right)\right| & =\left|s B\left(z_{0}\right)+C\left(z_{0}\right)\right| \\
& =\left|B\left(z_{0}\right)\right|\left|s+C\left(z_{0}\right) / B\left(z_{0}\right)\right| \\
& =\left|B\left(z_{0}\right)\right|\left|s+\mathfrak{R e}\left\{C\left(z_{0}\right) / B\left(z_{0}\right)\right\}+i \mathfrak{I m}\left\{C\left(z_{0}\right) / B\left(z_{0}\right)\right\}\right| \\
& \geq\left|B\left(z_{0}\right)\right|\left|1+\mathfrak{R e}\left\{C\left(z_{0}\right) / B\left(z_{0}\right)\right\}+i \mathfrak{I m}\left\{C\left(z_{0}\right) / B\left(z_{0}\right)\right\}\right| \\
& =\left|B\left(z_{0}\right)+C\left(z_{0}\right)\right|,
\end{aligned}
$$

which contradicts (2.11). Therefore, $|p(z)|<1$ in $\mathbb{D}$.

Theorem 2.4 Let $B(z)$ and $C(z)$ be analytic in $\mathbb{D}$ with

$$
\left|\mathfrak{I m}\left\{\frac{C(z)}{B(z)}\right\}\right| \geq \frac{1}{|B(z)|}, \quad z \in \mathbb{D} .
$$

If $p(z)$ is analytic in $\mathbb{D}$ with $p(0)=0$, and if

$$
\left|B(z) z p^{\prime}(z)+C(z) p(z)\right|<\sqrt{1+|B(z)|^{2}\left(\left|\frac{z p^{\prime}(z)}{p(z)}\right|+\mathfrak{R e}\left\{\frac{C(z)}{B(z)}\right\}\right)^{2}}, \quad z \in \mathbb{D},
$$

then we have

$$
|p(z)|<1, \quad z \in \mathbb{D} .
$$

Proof Applying the same method as in the proof of Theorem 2.3, we have

$$
\begin{aligned}
\left|B\left(z_{0}\right) z_{0} p^{\prime}\left(z_{0}\right)+C\left(z_{0}\right) p\left(z_{0}\right)\right| & =\left|p\left(z_{0}\right)\right|\left|B\left(z_{0}\right) \frac{z_{0} p^{\prime}\left(z_{0}\right)}{p\left(z_{0}\right)}+C\left(z_{0}\right)\right| \\
& =\left|s B\left(z_{0}\right)+C\left(z_{0}\right)\right|
\end{aligned}
$$




$$
\begin{aligned}
& =\left|B\left(z_{0}\right)\right|\left|s+C\left(z_{0}\right) / B\left(z_{0}\right)\right| \\
& =\left|B\left(z_{0}\right)\right|\left|s+\mathfrak{R e}\left\{C\left(z_{0}\right) / B\left(z_{0}\right)\right\}+i \mathfrak{I m}\left\{C\left(z_{0}\right) / B\left(z_{0}\right)\right\}\right| \\
& =\left|B\left(z_{0}\right)\right| \sqrt{\left(s+\mathfrak{R e}\left\{\frac{C\left(z_{0}\right)}{B\left(z_{0}\right)}\right\}\right)^{2}+\left(\mathfrak{I m}\left\{\frac{C\left(z_{0}\right)}{B\left(z_{0}\right)}\right\}\right)^{2}} .
\end{aligned}
$$

By (2.14), we have

$$
\begin{aligned}
\left|B\left(z_{0}\right) z_{0} p^{\prime}\left(z_{0}\right)+C\left(z_{0}\right) p\left(z_{0}\right)\right| & \geq\left|B\left(z_{0}\right)\right| \sqrt{\left(s+\mathfrak{R e}\left\{\frac{C\left(z_{0}\right)}{B\left(z_{0}\right)}\right\}\right)^{2}+\frac{1}{\left|B\left(z_{0}\right)\right|^{2}}} \\
& =\sqrt{1+\left|B\left(z_{0}\right)\right|^{2}\left(s+\mathfrak{R e}\left\{C\left(z_{0}\right) / B\left(z_{0}\right)\right\}\right)^{2}} \\
& =\sqrt{1+\left|B\left(z_{0}\right)\right|^{2}\left(\left|\frac{z_{0} p^{\prime}\left(z_{0}\right)}{p\left(z_{0}\right)}\right|+\mathfrak{R e}\left\{\frac{C\left(z_{0}\right)}{B\left(z_{0}\right)}\right\}\right)^{2}},
\end{aligned}
$$

which contradicts (2.15). Therefore, $|p(z)|<1$ in $\mathbb{D}$.

Remark 2.2 Theorem 2.3 and Theorem 2.4 improve a result obtained by Miller and Mocanu [5, p.206].

Theorem 2.5 Let $p(z)$ be analytic in $\mathbb{D}$ with $p(0)=1$ and

$$
\mathfrak{R e}\left\{2 p(z)-z p^{\prime \prime}(z) / p^{\prime}(z)-1\right\}>\alpha, \quad z \in \mathbb{D} .
$$

Then we have

$$
\mathfrak{R e}\{p(z)\}>\alpha, \quad z \in \mathbb{D}
$$

where $\alpha<1$.

Proof Putting $q(z)=(p(z)-\alpha) /(1-\alpha), q(0)=1$, we have to prove that $\mathfrak{R e}\{q(z)\}>0$ for $z \in \mathbb{D}$. If (2.18) does not hold, then

$$
q(z) \nprec \psi(z)=(1+z) /(1-z), \quad z \in \mathbb{D} .
$$

Hence by Lemma 1.1 there exist points

$$
z_{0}=r_{0} e^{i \theta} \in \mathbb{D} \quad \text { and } \quad \zeta_{0}, \quad\left|\zeta_{0}\right|=1
$$

for which

$$
\mathfrak{R e}\{q(z)\}>0 \quad \text { for }|z|<r_{0} \quad \text { and } \quad \mathfrak{R e}\left\{q\left(z_{0}\right)\right\}=\mathfrak{R e}\left\{\frac{1+\zeta_{0}}{1-\zeta_{0}}\right\}=0
$$

and

$$
z_{0} q^{\prime}\left(z_{0}\right)=s \zeta_{0} \psi^{\prime}\left(\zeta_{0}\right)=\frac{2 s \zeta_{0}}{\left(1-\zeta_{0}\right)^{2}}
$$


for some $s \geq 1$. Then, by (1.6), we have

$$
\mathfrak{R e}\left\{\frac{z_{0} q^{\prime \prime}\left(z_{0}\right)}{q^{\prime}\left(z_{0}\right)}+1\right\} \geq s \mathfrak{R e}\left\{\frac{\zeta_{0} \psi^{\prime \prime}\left(\zeta_{0}\right)}{\psi^{\prime}\left(\zeta_{0}\right)}+1\right\}=s \mathfrak{R e} \frac{1+\zeta_{0}}{1-\zeta_{0}}=0 .
$$

Therefore, we have

$$
\begin{aligned}
\mathfrak{R e} & \left\{2 p\left(z_{0}\right)-z_{0} p^{\prime \prime}\left(z_{0}\right) / p^{\prime}\left(z_{0}\right)-1\right\} \\
\quad= & \mathfrak{R e}\left\{2\left((1-\alpha) q\left(z_{0}\right)+\alpha\right)-z_{0} q^{\prime \prime}\left(z_{0}\right) / q^{\prime}\left(z_{0}\right)-1\right\} \\
\leq & \mathfrak{R e}\left\{2\left((1-\alpha) q\left(z_{0}\right)+\alpha\right)\right\} \\
\quad & \alpha,
\end{aligned}
$$

which contradicts (2.18). This completes the proof.

Remark 2.3 Theorem 2.5 improves a result obtained by Miller and Mocanu [5, p.207].

\section{Competing interests}

The authors declare that they have no competing interests.

\section{Authors? contributions}

All authors jointly worked on the results and they read and approved the final manuscript.

\section{Author details}

${ }^{1}$ University of Gunma, Hoshikuki-cho 798-8, Chuou-Ward, Chiba, 260-0808, Japan. ${ }^{2}$ Department of Applied Mathematics, College of Natural Sciences, Pukyong National University, Pusan, 608-737, Korea. ${ }^{3}$ Department of Mathematics, Kyungsung University, Busan, 608-736, Korea. ${ }^{4}$ Department of Mathematics, Rzeszów University of Technology, Al. Powstańców Warszawy 12, Rzeszów, 35-959, Poland.

\section{Acknowledgements}

The authors would like to express their thanks to the referees for their valuable advice regarding a previous version of this paper. This research was supported by the Basic Science Research Program through the National Research Foundation of Korea (NRF) funded by the Ministry of Education, Science and Technology (No. 2011-0007037).

Received: 3 November 2014 Accepted: 17 December 2014 Published online: 09 January 2015

\section{References}

1. Study, E: Konforme Abbildung einfach-zusammenhängender Bereiche. Teubner, Leipzig (1913)

2. Robertson, MS: On the theory of univalent functions. Ann. Math. 37, 374-408 (1936)

3. Miller, SS, Mocanu, PT: Differential subordinations and univalent functions. Mich. Math. J. 28, 151-171 (1981)

4. Miller, SS, Mocanu, PT: Differential Subordinations: Theory and Applications. Series of Monographs and Textbooks in Pure and Applied Mathematics, vol. 225. Dekker, New York (2000)

5. Miller, SS, Mocanu, PT: Differential subordination and inequalities in the complex plane. J. Differ. Equ. 67(2), 199-211 (1987)

6. Sokół, J, Nunokawa, M: On the subordination under Bernardi operator. Proc. Jpn. Acad., Ser. A, Math. Sci. 89, 11-14 (2013)

7. Nunokawa, M: On properties of non-Carathéodory functions. Proc. Jpn. Acad., Ser. A, Math. Sci. 68(6), 152-153 (1992)

8. Nunokawa, M: On the order of strongly starlikeness of strongly convex functions. Proc. Jpn. Acad., Ser. A, Math. Sci. 69(7), 234-237 (1993) 